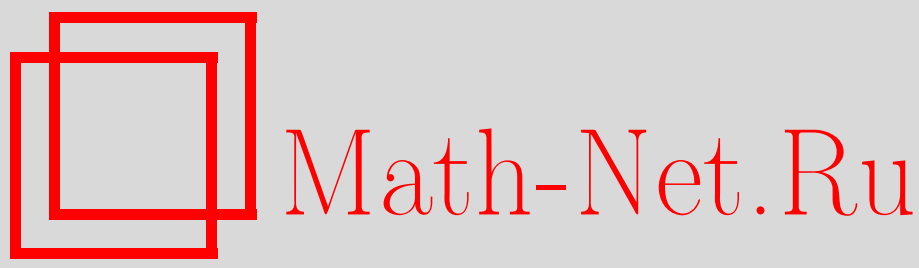

А. В. Маринов, Константы Липшица оператора метрического $\varepsilon$-проектирования в пространствах с заданными модулями выпуклости и гладкости, Изв. РАН. Сер. матем., 1998, том 62, выпуск 2, 103-130

DOI: https://doi.org/10.4213/im175

Использование Общероссийского математического портала Math-Net.Ru подразумевает, что вы прочитали и согласны с пользовательским соглашением

http://www . mathnet.ru/rus/agreement

Параметры загрузки:

IP: 54.92 .164 .108

26 апреля 2023 г., 13:02:38 
УДК 517.982.256

\author{
А. В. Маринов
}

\title{
Константы Липшица оператора метрического $\varepsilon$-проектирования в пространствах с заданными модулями выпуклости и гладкости
}

\begin{abstract}
Найдены неулучшаемые на классе всех линейных нормированных пространств оценки сверху для констант Липшица оператора метрического $\varepsilon$-проектирования $P$ через модули выпуклости и гладкости пространства при вариации трех параметров: приближаемого элемента $x$, выпуклого аппроксимирующего множества $M$ и точности аппроксимации $\varepsilon>0$. На их основе получены новые оценки устойчивости непрерывного селектора оператора $P$.

Библиография: 28 наименований.
\end{abstract}

\section{§1. Введение и основной результат}

В дальнейшем, если не оговорено противное, через $X$ обозначается линейное нормированное пространство над полем действительных чисел с нормой $\|\cdot\|$. Через $B(x, r), S(x, r)$ и $S$ обозначим шар $\{y \in X:\|x-y\| \leqslant r\}$, сфреру $(=r)$ и единичную сфреру $S(0,1)$ соответственно. Под $2^{X}$ понимается множество всех подмножеств из $X$.

Для непустого подмножества $M \subset X$, элемента $x \in X$ и $\varepsilon \geqslant 0$ положим

$$
x M=\inf \{\|x-z\|: z \in M\}
$$

- величина наилучшего приближения элемента $x$ множеством $M$ и

$$
x_{M}^{\varepsilon}=\{z \in M:\|x-z\| \leqslant x M+\varepsilon\}
$$

- метрическая в-проекиия $x$ на $M$.

Пусть $\mathscr{V}=\mathscr{V}(X)$ - множество тех троек $(x, M, \varepsilon)$, для которых $x_{M}^{\varepsilon} \neq \varnothing$. Оператор $P: \mathscr{V} \mapsto 2^{X} \backslash\{\varnothing\}$, задаваемый равенством

$$
P(v)=x_{M}^{\varepsilon}, \quad v=(x, M, \varepsilon),
$$

называется оператором метрического є-проектирования. Эти определения естественным образом обобщают классические понятия метрической проекции $x_{M}=$ $x_{M}^{0}$ и оператора метрического проектирования $x \mapsto x_{M}$ на заданное множество $M$.

Работа выполнена при финансовой поддержке Российского фонда фундаментальных исследований (грант № 93-0110-196).

(C) А.В. МАринов, 1998 
Метрику $\varrho$ на множестве $\mathscr{V}$ определим формулой

$$
\varrho(v, w)=|\varepsilon-\delta|+2\|x-y\|+3 \alpha(M, N),
$$

где $v=(x, M, \varepsilon), w=(y, N, \delta) \in \mathscr{V}, \alpha(M, N)$ - хаусдорфово расстояние между множествами $M$ и $N$, т.е.

$$
\begin{aligned}
& \alpha(M, N)=\max (\beta(M, N), \gamma(M, N)), \\
& \beta(M, N)=\gamma(N, M)=\sup _{p \in M} \inf _{q \in N}\|p-q\| .
\end{aligned}
$$

Пространство образов $2^{X} \backslash\{\varnothing\}$ оператора $P$ будем наделять одним из трех уклонений $\tau=\alpha, \beta, \gamma$. Введенные $\tau$-уклонения, как и расстояние $\varrho$ на $\mathscr{V}$, удовлетворяют неравенству треугольника: $\tau(A, B) \leqslant \tau(A, C)+\tau(C, B)$ для $A, B, C \subset X$, а хаусдорфово расстояние $\alpha$ вместе с метрикой $\varrho$ еше и симметричны. В дальнейшем используются сокращения $x y=\|x-y\|, M N=\beta(M, N)$, при этом выражения $2 x y, 3 M N$ и т.д. понимаются как $2\|x-y\|, 3 \beta(M, N)$ и т.д. Через $\operatorname{Cv}(X)$ обозначается множество всех непустых выпуклых подмножеств из пространства $X$.

$\mathrm{B}[1],[2]$ для $x \in X, M \in \mathrm{Cv}(X)$ и $\varepsilon>0$ при $\tau=\alpha, \beta, \gamma$ доказана $\tau$-липшицевость оператора $P$ в точке $v=(x, M, \varepsilon)$ по совокупности переменных, т.е. фактически установлена конечность и получены некоторые оценки сверху (см. (1.2), (1.1)) для $\tau$-констант Липшица $K_{\tau}(v)$, задаваемых формулами

$$
K_{\tau}(v)=\sup \left\{\frac{\tau(P(w), P(v))}{\varrho(v, w)}: w \in \mathscr{V}, \varrho(v, w) \neq 0\right\} .
$$

В настоящей статье, в частности, получены оценки сверху для $K_{\tau}(v)$ через модули выпуклости и гладкости пространства $X$ и показана их неулучшаемость на классе всех линейных нормированных пространств.

Устойчивости и оценкам устойчивости оператора $P$ при $\varepsilon>0$ посвящены работы [1]-[10] (более подробный обзор этих и других результатов можно найти в [2]). Заметим, что устойчивость оператора наилучшего приближения $x \mapsto x_{M}$ изучалась гораздо интенсивнее (см. [11]-[13]).

В дальнейшем нам будет удобнее иметь дело не с $\tau$-константами Липшица в точке $v$, а с некоторыми равномерными $\tau$-константами $K_{\tau}(a, s)$ и $K_{\tau}^{\prime}(a, s)$, которые определим следуюшим образом. При $a, s \geqslant 0$ положим

$$
\begin{aligned}
\mathscr{V}(a, s)=\{v= & (x, M, \varepsilon) \in \mathscr{V}: x \in X, M \in \operatorname{Cv}(X), x M \leqslant a, \varepsilon \geqslant s\}, \\
\mathscr{V}^{\prime}(a, s)=\{v= & (x, M, \varepsilon) \in \mathscr{V}: x \in X, M \in \operatorname{Cv}(X), x M=a, \varepsilon=s\}, \\
& K_{\tau}(a, s)=\sup \left\{K_{\tau}(v): v \in \mathscr{V}(a, s)\right\}, \\
& K_{\tau}^{\prime}(a, s)=\sup \left\{K_{\tau}(v): v \in \mathscr{V}^{\prime}(a, s)\right\} .
\end{aligned}
$$

Ясно, что

$$
\begin{gathered}
K_{\tau}^{\prime}(a, s) \leqslant K_{\tau}(a, s), \quad \mathscr{V}(a, s)=\cup\left\{\mathscr{V}^{\prime}(b, t): b \leqslant a, t \geqslant s\right\}, \\
K_{\tau}(a, s)=\sup \left\{K_{\tau}^{\prime}(b, t): b \leqslant a, t \geqslant s\right\} .
\end{gathered}
$$


В терминах равномерных констант проще формулируются утверждения о точности оценок сверху для $\tau$-констант Липшица (см., например, (1.9)); в то же время равномерные и поточечные оценки легко вытекают одна из другой (ср. оценки $(1.7),(1.8)$ с (2.27) и (3.19)).

Очевидны соотношения

$$
K_{\alpha}(v)=\max \left(K_{\beta}(v), K_{\gamma}(v)\right), \quad K_{\alpha}(a, s)=\max \left(K_{\beta}(a, s), K_{\gamma}(a, s)\right)
$$

и аналогичные для констант $K^{\prime}$.

Функция

$$
\varphi(t)=\inf \left\{1-\frac{\|x+y\|}{2}: x y \geqslant t, x, y \in S\right\}
$$

определенная для $t \in[0,2]$, называется модулем выпуклости пространства $X$. Пространство $X$ назьвается равномерно выпуклым. если $\varphi(t)>0$ для любого $t \in(0,2]$. Известно [14], [15], что $\varphi(t)$ строго возрастает на промежутке [ $\left.t_{0}, 2\right]$, где $t_{0}=\max \{t \in[0,2]: \varphi(t)=0\}$ и непрерывна на [0,2), поэтому обратную функцию естественно определить следуюшим образом:

$$
\varphi^{-1}(s)=\sup \left\{x y: 1-\frac{\|x+y\|}{2} \leqslant s, x, y \in S\right\} .
$$

Ясно, что $\varphi^{-1}$ возрастает и непрерывна на $[0,1]$. Для $a, s \geqslant 0, a+s>0$ положим

$$
\Phi(a, s)=(a+s) \varphi^{-1}\left(\frac{s}{a+s}\right)
$$

Из оценок устойчивости оператора $P$, полученных в теоремах 3,4 из [2], легко вытекают следуюшие оценки сверху для $\tau$-констант Липшица:

$$
\begin{aligned}
& K_{\beta}(a, s) \leqslant \frac{\Phi(a, s)}{s}-1 \leqslant \frac{2 a}{s}+1, \\
& K_{\alpha}(a, s) \leqslant \min \left(\frac{2 a}{s}+2,2 \frac{\Phi(a, s)}{s}\right) .
\end{aligned}
$$

Заметим, что в равномерно выпуклых пространствах $X$ оценки сверху для $K_{\tau}(a, s)$ через функцию $\Phi(a, s)$ при $a>0$ указывают на более медленный порядок роста к бесконечности констант Липшица при $s \rightarrow 0$, нежели оценка в общих пространствах $(\leqslant 2 a / s+1$ или $\leqslant 2 a / s+2)$. В самом деле, нетрудно показать (см. ниже лемму 9, в)), что справедливо порядковое равенство

$$
\frac{\Phi(a, s)}{s}-1 \asymp \frac{\varphi^{-1}(s)}{s}, \quad s \rightarrow 0 .
$$

Таким образом, в первом случае порядок не вьше, чем у отношения $\varphi^{-1}(s) / s$, причем $\varphi^{-1}(s) \rightarrow 0$ при $s \rightarrow 0$, а во втором - не выше, чем $1 / s$. 
Как отмечено в [2], в случае гильбертова пространства $X$ оценки через $\Phi$ дают правильный порядок роста, т.е. при $a>0$ и $\tau=\alpha, \beta, \gamma$ справедливы соотношения

$$
K_{\tau}(a, s) \asymp \frac{\varphi^{-1}(s)}{s} \asymp \frac{1}{\sqrt{s}}, \quad s \rightarrow 0 .
$$

В то же время в пространствах, единичная сфера $S$ которых содержит отрезок длины 2 , при $\tau=\alpha, \beta, \gamma$ выполняются порядковые равенства

$$
K_{\tau}(a, s) \asymp \frac{1}{s}, \quad s \rightarrow 0 .
$$

В этом нас убеждает справедливая в таких пространствах оценка снизу

$$
\frac{2 a}{s}+1 \leqslant K_{\tau}(a, s), \quad \tau=\alpha, \beta, \gamma
$$

(она вытекает из [2, $\S 3$, пример 1]), которая, с учетом (1.2) и (1.1), с одной стороны, дает равенства

$$
K_{\beta}(a, s)=\frac{\Phi(a, s)}{s}-1=\frac{2 a}{s}+1,
$$

а с другой - двусторонние оценки

$$
\frac{2 a}{s}+1 \leqslant K_{\tau}(a, s) \leqslant \frac{2 a}{s}+2, \quad \tau=\gamma, \alpha .
$$

Равенства (1.4) указывают на неулучшаемость оценки (1.1) на классе всех линейных нормированных пространств. Главная цель настоящей статьи состоит в получении неулучшаемых в том же смысле оценок для $K_{\tau}(a, s), \tau=\gamma, \alpha$, и, в частности, в ликвидации разрыва в оценках (1.5), а также в уточнении оценки (1.1) с использованием модуля гладкости пространства.

Чтобы сформулировать основной результат статьи - теорему 1 - введем еще одну величину

$$
\Psi(a, s)=\Phi(a, s)-\max \left[s, a \theta^{-1}\left(\frac{s}{2 a}\right)\right] .
$$

Здесь

$$
\theta(t)=\sup \left\{\frac{\|x+t y\|+\|x-t y\|}{2}-1: x, y \in S\right\}
$$

- модуль гладкости пространства $X$. Так как $\sqrt{1+t^{2}}-1 \leqslant \theta(t) \leqslant t$ и $\theta(t)-$ вьпуклая функция (см., например, [16]), то функция $\theta(t)$, а вместе с ней и обратная функция $\theta^{-1}(t)$ строго возрастают и непрерывны при $t \geqslant 0$. В идейном отношении появление величины $\Psi(a, s)$ связано с леммой 1 из [17]. Ясно, что

$$
\Psi(a, s) \leqslant \Phi(a, s)-s \leqslant 2 a+s .
$$


TЕОРема 1. При $а \geqslant 0, s>0$ справедливы оценки

$$
\begin{aligned}
& K_{\beta}(a, s) \leqslant \frac{\Psi(a, s)}{s} \leqslant \frac{2 a}{s}+1, \\
& K_{\alpha}(a, s) \leqslant \frac{\Phi(a, s)}{s}-1 \leqslant \frac{2 a}{s}+1 .
\end{aligned}
$$

А если дополнительно предположить, что единичная сфера пространства $X$ содержит отрезок длины 2 , то при $\tau=\alpha, \beta, \gamma$ имеем

$$
K_{\tau}^{\prime}(a, s)=K_{\tau}(a, s)=\frac{2 a}{s}+1
$$

Заметим, что равенства (1.9) выполняются и в некоторых строго выпуклых пространствах, т.е. таких пространствах, в которых единичная сфера $S$ вообще не содержит отрезков. Таково, например, построенное в [15] строго выпуклое пространство с разрывным модулем выпуклости: $\varphi(t)=0$ при $t \in[0,2)$ и $\varphi(2)=1$.

Доказательству оценки (1.7) посвящен $\S 2$, а $(1.8)-\S 3$. При этом получены новые оценки устойчивости оператора $P$, уточняющие теоремы 3,4 из [2]. Равенства (1.9) вытекают из (1.7), (1.8) и (1.3). В $\S 4$ даются уточнения полученных результатов для $\alpha$-устойчивости при дополнительном предположении выпуклости множества $N$, что в свою очередь используется в $\S 5$ для оценок устойчивости непрерывного селектора оператора $P$. В $\S 6$ показано, что оценки устойчивости оператора $P$, полученные в $\S 2,3$, позволяют получить неулучшаемые оценки $\tau$-констант Липшица оператора $P$ не только по совокупности переменных $x, M, \varepsilon$, но и по каждой из переменных в отдельности.

Как уже отмечалось, оценки (1.3) вытекают из [2, §3, пример 1], где показано, что в двумерном пространстве $X$ с квадратом в качестве единичной сферы $S$ для любых $a \geqslant 0, \delta \geqslant \varepsilon \geqslant 0$ сушествуют точка $x \in X$ и прямая $M$ такие, что $x M=a$ и

$$
x_{M}^{\delta} x_{M}^{\varepsilon}=\left(\frac{2 a}{\delta}+1\right)(\delta-\varepsilon) .
$$

Отсюда следует, что при $\tau=\alpha, \beta, \gamma$ через величину $2 a / s+1$ оцениваются снизу соответствующие равномерные $\tau$-константы Липшища оператора $P$ по переменной $\varepsilon$ (т.е. при вариации только одного параметра $\varepsilon$ ), а значит, и константы $K_{\tau}(a, s)$. Попутно установлено, что теорема 1 останется справедливой, если в ней заменить $K_{\tau}(a, s)$ на равномерные $\tau$-константы Липшица по переменной $\varepsilon$. В $\S 6$ аналогичный результат получен для констант Липшица по переменным $x$ и $M$ в отдельности.

Заметим, что в [2], вслед за более ранними исследованиями (см. [6] и библиографию к ней), рассмотрена более общая ситуация, когда варьируется еще и норма пространства $X$. Здесь мы ограничимся только тремя переменными $x, M, \varepsilon$. Темне менее полезно отметить, что часть результатов статьи автоматически переносится на общий случай (см. замечание в конще $\S 2)$.

Всюду в дальнейшем множества $M, N$ и проекции $x_{M}^{\varepsilon}, y_{N}^{\delta}$ предполагаются непустыми. Будем пользоваться сокращениями вида

$$
x_{M}^{\varepsilon} x_{M}^{+0}=\lim _{t \rightarrow+0} x_{M}^{\varepsilon} x_{M}^{t}, \quad x_{M}^{\varepsilon} y_{N}^{\delta-0}=\lim _{t \rightarrow+0} x_{M}^{\varepsilon} y_{N}^{\delta-t}
$$


и т.д. Для непустых множеств $A, B \subset X$ положим

$$
\chi(A, B)=\sup \{x, y: x \in A, y \in B\} .
$$

Диаметр подмножества $A \subset X$ обозначим через $\operatorname{diam} A$.

\section{§2. Оценки для $\beta$-констант Липшица и промежуточные неравенства}

Оценка (1.7) для $K_{\beta}(a, s)$ будет получена последовательным доказательством следуюшей цепочки неравенств:

$$
K_{\beta}(a, s) \leqslant \frac{\xi(a, s)}{s} \leqslant \frac{\zeta(a, s)}{s} \leqslant \frac{\Psi(a, s)}{s},
$$

где

$$
\begin{aligned}
& \xi(a, s)=\sup \left\{x_{M}^{s} x_{M}^{+0}: x \in X, M \in \operatorname{Cv}(X), x M=a\right\}, \\
& \zeta(a, s)=\sup \left\{\chi\left(x_{M}^{s} x_{M}^{+0}\right): x \in X, M \in \operatorname{Cv}(X), x M=a\right\} .
\end{aligned}
$$

Введение промежуточных величин $\xi(a, s)$ и $\zeta(a, s)$ объясняется тем, что, во-первых, $\xi(a, s)$ (как, впрочем, и $\zeta(a, s))$ обладает необходимыми свойствами монотонности, существенно используюшимися при доказательстве первого неравенства в $(2.1)$, и, во-вторых, тем, что величина $\zeta(a, s)$ допускает более простое представление, позволяющее установить последнее неравенство (заметим, что среднее неравенство в (2.1) тривиально). Аналогичные свойства монотонности для функции $\Psi(a, s)$ установить не удается.

Доказательство (2.1) начнем с первого неравенства. Из [2, лемма 10] следует, что для любых $v=(x, M, \varepsilon), w=(y, N, \delta) \in \mathscr{V}$ с выпукльгм $M$ и

$$
\lambda=\max (\varepsilon, \delta)+2 x y+2 \alpha(M, N)>0
$$

справедлива оценка

$$
y_{N}^{\delta} x_{M}^{\varepsilon} \leqslant N M+\frac{x_{M}^{\lambda} x_{M}^{+0}}{\lambda}(|\delta-\varepsilon|+2 x y+2 \alpha(M, N)) .
$$

При этом в [2] (см. (3.8)) установлена также монотонность отношения $x_{M}^{\lambda} x_{M}^{+0} / \lambda$, а именно

$$
\frac{x_{M}^{\delta} x_{M}^{+0}}{\delta} \leqslant \frac{x_{M}^{\varepsilon} x_{M}^{+0}}{\varepsilon}
$$

если $\delta \geqslant \varepsilon$. Так как $\varepsilon \leqslant \lambda$, то из этих результатов легко следует оценка

$$
K_{\beta}(v) \leqslant \max \left(1, \frac{x_{M}^{\varepsilon} x_{M}^{+0}}{\varepsilon}\right) .
$$

Беря в качестве $M$ прямую, опорную к шару $B(x, a)$, без труда убеждаемся в том, что $x_{M}^{\varepsilon} x_{M}^{+0} \geqslant \varepsilon$, значит,

$$
\xi(a, s) \geqslant s
$$


Поэтому из (2.4) следует неравенство

$$
K_{\beta}^{\prime}(a, \varepsilon) \leqslant \frac{\xi(a, \varepsilon)}{\varepsilon}
$$

Далее нам понадобятся следуюшие усиления соотношения (2.3).

ЛЕмма 1 [2]. Пусть $x \in X, M$ выпукло, $\delta \geqslant \varepsilon>t>0\left(t \geqslant 0\right.$ при $\left.x_{M} \neq \varnothing\right)$, u пусть $G \subset x_{M}^{t}$. Тогда

$$
\frac{x_{M}^{\delta} G}{\delta-t} \leqslant \frac{x_{M}^{\varepsilon} G}{\varepsilon-t}
$$

Лемма 2 [2]. Пусть $x \in X, M$ выпукло, $\delta>\varepsilon>0, t>s>0(\varepsilon, s \geqslant 0$ при $\left.x_{M} \neq \varnothing\right)$, и пусть $\delta \geqslant t, \varepsilon \geqslant s$. Тогда

$$
\frac{x_{M}^{\delta} x_{M}^{\varepsilon}}{\delta-\varepsilon} \leqslant \frac{x_{M}^{t} x_{M}^{s}}{t-s}
$$

СлЕДСТвиЕ 1 [1]. При $\delta>\varepsilon>0$ имеем

$$
x_{M}^{\delta} x_{M}^{\varepsilon} \leqslant \frac{x_{M}^{\delta} x_{M}^{+0}}{\delta}(\delta-\varepsilon) .
$$

ЛЕмма 3. Функция $\xi(a, s)$ возрастает по обоим аргументам в области $a \geqslant 0, s>0$, а отношение $\xi(a, s) / s$ убьввает по переменной $s$.

ДокАЗАТЕЛЬСтво. Пусть $0 \leqslant a \leqslant b, 0<s \leqslant t$. Покажем неравенство

$$
\xi(a, s) \leqslant \xi(b, t)
$$

Пусть $x \in X, M$ выпукло, причем $x M=a$. Рассмотрим гомотетию $f(y)=$ $a(y-x) / b+x$ пространства $X$ с центром в точке $x$ и коэффициентом $a / b$. Пусть $L=f(M)$. Ясно, что $x L=b$ и $L$ выпукло. Кроме того, если положить $\varepsilon=a s / b$, $\delta=a l / b$, то, очевидно, $0<l<s$ и

$$
\frac{b}{a} x_{M}^{\varepsilon} x_{M}^{\delta}=x_{L}^{s} x_{L}^{l}
$$

Отсюда ввиду (2.8) имеем

$$
x_{M}^{s} x_{M}^{l}=\frac{x_{M}^{\varepsilon} x_{M}^{\delta}}{\varepsilon-\delta}(s-l)=\frac{b}{a} x_{M}^{\varepsilon} x_{M}^{\delta}=x_{L}^{s} x_{L}^{l} \leqslant x_{L}^{t} x_{L}^{l} .
$$

Переходя здесь к пределу при $l \rightarrow 0$ и взяв затем верхнюю грань по всем $M \in \mathrm{Cv}(X)$ с $x M=a$, получаем (2.10).

Монотонность отношения $\xi(a, s) / s$ доказывается переходом к верхней грани по всем выпуклым множествам $M \subset X$ с $x M=a$ в неравенстве (2.3). 
Лемма 4. Пусть $a \geqslant 0, s>0$. Тогда

$$
K_{\beta}(a, s) \leqslant \frac{\xi(a, s)}{s} .
$$

ДокАЗАТЕЛЬСТво. Пользуясь неравенством (2.6) и предыдущей леммой, имеем

$$
K_{\beta}(a, s)=\sup _{\substack{b \leqslant a \\ \varepsilon \geqslant s}} K_{\beta}^{\prime}(b, \varepsilon) \leqslant \sup _{\substack{b \leqslant a \\ \varepsilon \geqslant s}} \frac{\xi(b, \varepsilon)}{\varepsilon} \leqslant \sup _{\varepsilon \geqslant s} \frac{\xi(a, \varepsilon)}{\varepsilon} \leqslant \frac{\xi(a, s)}{s} .
$$

Следуюшая лемма показывает, что величина $\zeta(a, s)$ обладает теми же свойствами монотонности, что и $\xi(a, s)$ (этот факт не используется в дальнейшем, но, по-видимому, представляет самостоятельный интерес).

ЛЕмма 5. Функция $\zeta(a, s)$ монотонно возрастает по обоим аргументам в области $a \geqslant 0, s>0$, а отношение $\zeta(a, s) / s$ монотонно убывает по $s$.

ДокАЗАТЕЛЬСтво. Полагая в (2.7) $G=y \in x_{M}^{t}$ и переходя затем к верхней грани по $y \in x_{M}^{t}$, получаем следующую оценку:

$$
\frac{\chi\left(x_{M}^{\delta}, x_{M}^{t}\right)}{\delta-t} \leqslant \frac{\chi\left(x_{M}^{\varepsilon}, x_{M}^{t}\right)}{\varepsilon-t}
$$

при $\delta \geqslant \varepsilon>t>0$. Устремляя здесь $t$ к нулю и взяв затем верхнюю грань по всем $x \in X$ и $M \in \mathrm{Cv}(X)$ таким, что $x M=a$, приходим к нужному неравенству $\zeta(a, \delta) / \delta \leqslant \zeta(a, \varepsilon) / \varepsilon$.

Доказательство монотонности функции $\zeta(a, s)$ по обеим переменным дословно повторяет доказательство для $\xi(a, s)$ с той лишь разницей, что все $\beta$-уклонения в последнем нужно заменить на $\chi$-расстояния, а вместо неравенства (2.8) использовать (2.12).

Лемма 6. Пусть $x \in X, \quad M \in \operatorname{Cv}(X), \varepsilon \geqslant t>0 \quad\left(t \geqslant 0\right.$ nрu $\left.x_{M} \neq \varnothing\right)$, $x M>0, H$ - гиперплоскость, разделяющая шар $B(x, x M)$ и множество $M$. Тогда

$$
\chi\left(x_{M}^{\varepsilon}, x_{M}^{t}\right) \leqslant \chi\left(x_{H}^{\varepsilon}, x_{H}^{t}\right) .
$$

ДокаЗАТЕЛЬСтво. Пусть $y \in x_{M}^{\varepsilon}, z \in x_{M}^{t}, y \neq z$. Проведем луч $l=\{\alpha z+$ $(1-\alpha) x: \alpha \geqslant 0\}$. Пусть $v$ - точка пересечения $l$ и сферы $S(x, x M+\varepsilon)$. Пусть Пдвумерная плоскость, проходящая через точки $x, y, v$ (при $y \in l$ берем одну из таких плоскостей). Обозначим $S_{2}=S(x, x M+\varepsilon) \cap \Pi$ сферу в плоскости $(\Pi,\|\cdot\|)$. Она разбивается на две дуги диаметрально противоположными точками $v$ и $2 x-v$.

Пусть точка $w \in S_{2}$ удаляется от точки $v$ по одной из двух дуг по направлению к точке $2 x-v$. Нетрудно показать, что при этом расстояние $z w$ не убывает. Отсюда следует, что одно из расстояний $z w_{i}, i=1,2$, где $w_{i} \in H \cap S_{2}$, не меньше расстояния между точками $y$ и $z$, скажем,

$$
y z \leqslant w_{1} z
$$


Из этих же соображений получаем, что расстояние от любой из двух точек пересечения $H \cap S(x, x z)$ до точки $p=l \cap H$ не меньше $p z$. Значит, если через $q$ обозначить ту из них, которая лежит между $p$ и $w_{2}$, то получим

$$
w_{1} z \leqslant w_{1} p+p z \leqslant w_{1} p+p q=w_{1} q .
$$

А так как $q \in x_{H}^{t}, w_{1} \in x_{H}^{\varepsilon}$, то из (2.14) и (2.15) ввиду произвольности точек $y \in x_{M}^{\varepsilon}$ и $z \in x_{M}^{t}$ следует (2.13).

СледСтвие 2. $\chi\left(x_{M}^{\varepsilon}, x_{M}^{+0}\right) \leqslant \chi\left(x_{H}^{\varepsilon}, x_{H}^{+0}\right)$.

Отсюда вытекает, что в определении величины $\zeta(a, s)$ верхнюю грань можно брать только по гиперплоскостям, т.е. получаем такое

СлЕДСТвИЕ 3 . При $a \geqslant 0, s>0$ справедливо представление

$$
\zeta(a, s)=\sup \left\{\chi\left(x_{H}^{s}, x_{H}^{+0}\right): x \in X, H-\text { гиперплоскость в } X, x H=a\right\} .
$$

В [18] для выпуклого $M$ получена оценка

$$
\operatorname{diam} x_{M}^{\varepsilon} \leqslant \Phi(x M, \varepsilon)
$$

а в [2] - неравенство

$$
x_{M}^{\varepsilon} x_{M}^{+0} \leqslant \Phi(x M, \varepsilon)-\varepsilon
$$

причем здесь $x_{M}^{+0}$ можно заменить на $x_{M}$, если $x_{M} \neq \varnothing$.

Следуюшая лемма уточняет оценку (2.17).

Лемма 7. Пусть $a \geqslant 0, s>0$. Тогдa

$$
\zeta(a, s) \leqslant \Psi(a, s)
$$

ДокАЗАТЕЛЬСтво. Ввиду следствия 2 из леммы 6 достаточно показать справедливость неравенства

$$
\chi\left(x_{H}^{s}, x_{H}^{+0}\right) \leqslant \Psi(a, s)
$$

для любой гиперплоскости $H$, опорной к шару $B(x, a)$. Пусть $p \in S(x, a+s)$, $0<t<s, z \in x_{H}^{t}, L-$ прямая, проходящая через точки $p$ и $z$, а $q$-вторая точка пересечения прямой $L$ и сферы $S(x, a+s)$. Имеем

$$
q z=q p-p z \leqslant \operatorname{diam} x_{H}^{s}-\inf \left\{p_{1} z_{1}: p_{1} \in S(x, x M+\varepsilon) \cap H, z \in x_{H}^{t}\right\} .
$$

Переходя в левой части к верхней грани по соответствующим $q$ и $z$ и обозначая последний inf через $g(t)$, с учетом (2.16) получаем

$$
\chi\left(x_{H}^{s}, x_{H}^{t}\right) \leqslant \Phi(x H, s)-g(t) .
$$

Ясно, что

$$
g(t) \geqslant s-t
$$


Для оценки $g(t)$ снизу через модуль гладкости пространства $X$ воспользуемся идеей доказательства леммы 1 из [17]. Будем считать, что $x=0$. Оценим расстояние $p z$ снизу.

Пусть $y=z /(a+s), u=(p-z) /(a+s)$. Тогда $\|y+u\|=1$, а значит,

$$
\left\|\frac{y}{\|y\|}+\frac{u}{\|y\|}\right\|=\frac{1}{\|y\|} \geqslant \frac{a+s}{\|z\|} \geqslant \frac{a+s}{(a+t)} .
$$

Кроме того, по построению $y \in H /(a+s)$, а вектор $u$ параллелен $H$, в то время как $H /(a+s)$ - гиперплоскость, опорная к $B(0, a /(a+s))$, поэтому точка $y-u$ лежит вне шара $B(0, a /(a+s))$ и, следовательно,

$$
\left\|\frac{y}{\|y\|}-\frac{u}{\|y\|}\right\| \geqslant \frac{a}{a+s} \cdot \frac{1}{\|y\|} \geqslant \frac{a}{a+t} .
$$

Таким образом,

$$
\begin{aligned}
\theta\left(\frac{\|u\|}{\|y\|}\right) & \geqslant \frac{1}{2}\left(\left\|\frac{y}{\|y\|}+\frac{u}{\|y\|}\right\|+\left\|\frac{y}{\|y\|}-\frac{u}{\|y\|}\right\|\right)-1 \\
& \geqslant\left(\frac{a+s}{2(a+t)}+\frac{a}{2(a+t)}\right)-1=\frac{s-2 t}{2(a+t)} .
\end{aligned}
$$

Отсюда

$$
\|u\| \geqslant\|y\| \theta^{-1}\left(\frac{s-2 t}{2(a+t)}\right)
$$

или

$$
p z \geqslant\|z\| \theta^{-1}\left(\frac{s-2 t}{2(a+t)}\right) \geqslant a \theta^{-1}\left(\frac{s-2 t}{2(a+t)}\right) .
$$

Поскольку такая же оценка снизу справедлива и для $g(t)$, то, принимая во внимание (2.20), получаем

$$
\chi\left(x_{H}^{s}, x_{H}^{t}\right) \leqslant \Phi(a, s)-\max \left[s-t, \theta^{-1}\left(\frac{s-2 t}{2(a+t)}\right)\right] .
$$

Переходя здесь к пределу при $t \rightarrow 0$ и учитьвая непрерьвность функции $\theta^{-1}$, приходим к (2.19). Лемма доказана.

Итак, доказательство цепочки неравенств (2.1), а вместе с ней и оценки (1.7), полностью завершено. Попутно в $\S 2$ получена новая оценка $\beta$-устойчивости оператора $P$, улучшаюшая оценку (4.5) теоремы 3 из [2] (в случае вариации трех параметров $x, M, \varepsilon)$. Сформулируем этот результат в виде теоремы. 
TеОРема 2. Пусть $M$ - выпуклое подмножество из $X, N \subset X, x, y \in X$, $\varepsilon, \delta \geqslant 0 u$

$$
\lambda=\max (\varepsilon, \delta)+2 x y+2 \alpha(M, N)>0 .
$$

Тогда

$$
y_{N}^{\delta} x_{M}^{\varepsilon} \leqslant N M+\frac{\Psi(x M, \lambda)}{\lambda}(|\delta-\varepsilon|+2 x y+2 \alpha(M, N)),
$$

причем $\lambda$ в этом неравенстве можно заменить на $\mu=\max (\varepsilon, \delta)$ или на $\varepsilon в$ случае их положительности.

Неравенство (2.22) следует из (2.2) и соотношения

$$
x_{M}^{t} x_{M}^{+0} \leqslant \Psi(x M, t), \quad t>0,
$$

а возможность замены $\lambda$ на $\mu$ или на $\varepsilon$ вытекает из того, что $\lambda \geqslant \mu \geqslant \varepsilon$, свойства монотонности $(2.3)$ и опять же $(2.23)$.

Приведем еще одно полезное для дальнейшего утверждение. Из леммы 6 , неравенства (2.21) и того, что в (2.21) $x M=x H=a$, вытекает следуюшая

Лемма 8. Если $M \in \operatorname{Cv}(X), x \in X, \varepsilon \geqslant t>0\left(t \geqslant 0\right.$ nрu $\left.x_{M} \neq \varnothing\right), m o$

$$
\chi\left(x_{M}^{\varepsilon}, x_{M}^{t}\right) \leqslant \Phi(x M, \varepsilon)-(\varepsilon-t) .
$$

В связи с соотношением (1.6) естественно возникает вопрос о справедливости аналога неравенства (1.7) из теоремы 1 для константы $K_{\alpha}(a, s)$, т.е. о справедливости неравенства

$$
K_{\alpha}(a, s) \leqslant \frac{\Psi(a, s)}{s},
$$

усиливающего оценку (1.8). И хотя ответ на этот вопрос автору не известен (естественно, за исключением тех пространств, в которых выполняются равенства (1.9)), но тем не менее в случае евклидова пространства $X=\mathbb{R}^{2}$ удается показать непригодность для доказательства (2.25) метода промежуточных оценок, использованного при получении неравенства (1.7). Оказывается, что в $\mathbb{R}^{2}$ для любых $a, s>0$ вьполняется соотношение

$$
K_{\alpha}(a, s)>\frac{\zeta(a, s)}{s} .
$$

При доказательстве (2.26) без ограничения обшности можно предположить, что $a=1$. Пусть $x \in \mathbb{R}^{2}, t>0, M$ и $L$ - параллельные прямые, касающиеся шаров $B(x, 1)$ и $B(x, 1+t)$ соответственно и отстоящие друг от друга на расстояние $t$. Через $q$ обозначим точку пересечения $L \cap B(x, 1+t)$, через $z$ - одну из точек пересечения $M \cap B(x, 1+t)$. Опустим из точки $z$ перпендикуляр на прямую $L$ : $p \in L,[p, z] \perp L$. Положим $N=[p, z] \cup(L \backslash\{q\})$. Тогда $\alpha(M, N)=N M=t$, $x_{N}=z$ и

$$
x_{M}^{s} x_{N}=\alpha\left(x_{M}^{s}, x_{N}\right)=\sqrt{s^{2}+2 s}+\sqrt{t^{2}+2 t} .
$$


Но $\zeta(a, \varepsilon)=\sqrt{\varepsilon^{2}+2 \varepsilon}$, поэтому для $v=(x, M, s)$ имеем

$$
\begin{aligned}
K_{\gamma}(1, s) & \geqslant K_{\gamma}(v)=\sup \left\{\frac{P(v) P(w)}{\varrho(v, w)}: w \in \mathscr{V}, \varrho(v, w) \neq 0\right\} \\
& \geqslant \sup _{t>0} \frac{x_{M}^{s} x_{N}}{s+3 t}>\frac{\sqrt{s^{2}+2 s}}{s}=\frac{\zeta(1, s)}{s} .
\end{aligned}
$$

В приведенном примере верхняя грань берется по точкам $w$ вида $(x, N, 0)$ при некотором незамкнутом множестве $N$. Нетрудно подправить пример таким образом, чтобы точка $w$ имела вид $(x, N, s / 2)$, а множество $N$ было замкнутым. Для этого нужно лишь продлить отрезок $[p, z]$ за точку $z$ до соответствующего отрезка $\left[p, z^{\prime}\right]$.

Полезно привести поточечный вариант неравенства (1.7). При $x \in X, M \in$ $\mathrm{Cv}(X)$ и $\varepsilon>0$ для точки $v=(x, M, \varepsilon)$ выполняются соотношения

$$
K_{\beta}(v) \leqslant \frac{\Psi(x M, \varepsilon)}{\varepsilon} \leqslant \frac{2 x M}{\varepsilon}+1 .
$$

В заключение заметим,что фактически в $\S 2$ доказано больше, а именно, что неравенства (1.7) и (2.27) справедливы и в случае вариации нормы пространства. Это становится вполне очевидньм, если при их доказательстве вместо неравенства (2.2) воспользоваться его обобщением - оценкой (4.1) леммы 10 из [2].

\section{§ 3. Оценка для $\alpha$-констант Липшица}

Ввиду уже доказанной оценки (1.7) и соотношений (1.6) для доказательства (1.8) достаточно получить неравенство

$$
K_{\gamma}(a, s) \leqslant \frac{\Phi(a, s)}{s}-1 .
$$

В этом нам поможет следующая

Теорема 3. Пусть $M$ - выпуклое подмножество из $X, N \subset X, z, y \in X$, $\varepsilon, \delta \geqslant 0, \mu=\max (\varepsilon, \delta)>0$. Тогда при $\tau=\alpha, \gamma$ справедливы неравенства

$$
\tau\left(y_{N}^{\delta}, x_{M}^{\varepsilon}\right) \leqslant \tau(N, M)+\left(\frac{\Phi(x M, \mu)}{\mu}-1\right)(|\delta-\varepsilon|+2 x y+2 \alpha(M, N)),
$$

причем $\mu$ здесь можно заменить на $\varepsilon$, если $\varepsilon>0$.

Заметим сразу, что при $\tau=\beta$ неравенство (3.2) уже установлено - оно является следствием более сильной оценки теоремы 2. Поэтому если мы докажем теорему 3 при $\tau=\gamma$, то тем самым докажем ее и при $\tau=\alpha$, т.е. останется рассмотреть только случай $\tau=\gamma$.

Неравенство (3.1) является тривиальным следствием оценки (3.2) при $\tau=\alpha$. Нужно только воспользоваться соотношением

$$
\frac{\Phi(x M, t)}{t}-1 \geqslant 1, \quad t>0,
$$

которое, наряду с другими свойствами функции $\Phi(a, s)$, содержится в следуюшей лемме. 
Лемма 9 [2]. а) Ecлu $a+t \leqslant b+s u t \leqslant s, m o$

$$
\Phi(a, t) \leqslant \Phi(b, s)
$$

в частности, функиия $\Phi(a, t)$ не убьвает по каждой переменной $a, t$.

б) Отношение $\Phi(a, t) / t$ не возрастает по $t$ при $t>0$.

в) При $a, t \geqslant 0$ справедливь оченки

$$
\begin{aligned}
\min (a+t, 1) \varphi^{-1}(t) & \leqslant \Phi(a, t) \leqslant \max (a+t, 1) \varphi^{-1}(t), \\
2 t & \leqslant \Phi(a, t) \leqslant 2(a+t) .
\end{aligned}
$$

Оценка (3.2) уточняет неравенство (4.6) из [2] при вариации трех параметров $x, M, \varepsilon$. Из (3.2) также легко следует неравенство

$$
\alpha\left(y_{N}^{\delta}, x_{M}^{\varepsilon}\right) \leqslant \frac{\Phi(x M, \mu)}{\mu}(|\delta-\varepsilon|+2 x y+2 \alpha(M, N)),
$$

где $\mu$ можно заменить на $\varepsilon>0$. Возможность замены $\mu$ на $\varepsilon$ в (3.2) и (3.3) вытекает из леммы 9, б). Оценка (3.4) улучшает результат следствия 2 теоремы 1 из [2].

Доказательство теоремы 3 разбивается на ряд случаев. В первом случае (грубо говоря, при малых возмушениях параметра $M$ ) нам понадобится оценка расстояния $x_{M}^{\varepsilon} y_{N}^{\delta}$ через величину вида $y_{M}^{t} y_{M}^{s}$ (см. ниже (3.10)). В работах [1], [2] в подобном случае использовалась оценка через величину вида $x_{M}^{t} x_{M}^{s}$, что приводило к менее точным оценкам устойчивости оператора $P$.

Определения метрической $\varepsilon$-проекции и $\tau$-расстояний в произвольном метрическом пространстве $X$ аналогичны их определениям в линейном нормированном пространстве.

Лемма 10. Пусть $X$ - метрическое пространство, $M, N, F \subset X, y \in X$, $\delta \geqslant 0$, и пусть выполняется условие

$$
y M+M N<\delta+y N
$$

Тогда величина $s=\delta+y N-y M-M N$ положительна и имеет место неравенство

$$
F y_{N}^{\delta} \leqslant M N+F y_{M}^{s-0} .
$$

ДокАЗАТЕЛЬСтво. Пусть $0<t<s$. По неравенству треугольника получаем

$$
F y_{N}^{\delta} \leqslant F y_{M}^{s-t}+y_{M}^{s-t} y_{N}^{\delta} .
$$

Покажем, что $y_{M}^{s-t} y_{N}^{\delta} \leqslant M N$, т.е., другими словами, что $z y_{N}^{\delta} \leqslant M N$ при $z \in y_{M}^{s-t}$. Имеем

$$
z y \leqslant y M+s-t=\delta+y N-M N-t .
$$

Ho

$$
z y_{N}^{\delta} \leqslant z z_{N}^{t}+z_{N}^{t} y_{N}^{\delta}
$$


и при этом, очевидно, $z z_{N}^{t} \leqslant z N \leqslant M N$. Остается показать, что второе слагаемое в (3.8) равно нулю.

Пусть $p \in z_{N}^{t}$. Тогда $p \in N$ и $p z \leqslant z N+t \leqslant M N+t$. Отсюда с учетом (3.8) имеем

$$
p y \leqslant p z+z y \leqslant M N+t+\delta+y N-M N-t=\delta+y N .
$$

Поэтому $p \in y_{N}^{\delta}$, т.е. $z_{N}^{t} \subset y_{N}^{\delta}$ и $z_{N}^{t} y_{N}^{\delta}=0$. Итак, $y_{M}^{s-t} y_{N}^{\delta} \leqslant M N$ и оценка (3.6) получается из (3.7) заменой второго слагаемого на $M N$ и последующим переходом к пределу при $t \rightarrow 0$.

СлЕДСТВИЕ 4. Пусть $t>0\left(t \geqslant 0\right.$, eсли $\left.x_{M} \neq \varnothing\right), l=t+x y+x M-y M$. Тогда в условиях леммы 10 выполняется неравенство

$$
x_{M}^{t} y_{N}^{\delta} \leqslant M N+y_{M}^{l} y_{M}^{s-0} .
$$

Для доказательства нужно только положить в (3.6) $F=x_{M}^{t}$ и заметить, что $x_{M}^{t} \subset y_{M}^{l}$.

ДоКАЗАТЕЛЬСТво ТЕОРЕМЫ 3. Как уже отмечалось, неравенство (3.2) достаточно установить только для $\tau=\gamma$. Докажем больше, а именно, что та же оценка сверху справедлива и для расстояния $x_{M}^{\mu} y_{N}^{\delta}$, где $\mu=\max (\varepsilon, \delta)$. Доказательство распадается на ряд случаев и подслучаев.

СлучАй 1. $y M+M N<\delta+y N$, т.е. выполняется условие (3.5) леммы 10. Воспользовавшись следствием из леммы 10 при $t=\mu$, получаем

$$
x_{M}^{\mu} y_{N}^{\delta} \leqslant M N+y_{M}^{l} y_{M}^{s-0},
$$

где

$$
\begin{aligned}
& l=\mu+y x+x M-y M, \\
& s=\delta+y N-y M-M N .
\end{aligned}
$$

Отсюда ввиду выпуклости $M$ и неравенства (2.9) имеем

$$
x_{M}^{\mu} y_{N}^{\delta} \leqslant M N+\frac{y_{M}^{l} y_{M}^{+0}}{l}(l-s) .
$$

Из неравенства треугольника легко следуют соотношения

$$
\begin{gathered}
0 \leqslant l-s \leqslant|\varepsilon-\delta|+2 x y+N M+M N, \\
\mu \leqslant l \leqslant \mu+2 x y .
\end{gathered}
$$

Кроме того, из условия $s>0$ вытекает

$$
0 \leqslant \frac{l-s}{l}<1 .
$$

Далее, ввиду (2.18) $y_{M}^{l} y_{M}^{+0} \leqslant \Phi(y M, l)-l$, а потому из (3.11) следует

$$
x_{M}^{\mu} y_{N}^{\delta} \leqslant M N+\left(\frac{\Phi(y M, l)}{l}-1\right)(l-s) .
$$

Рассмотрим два подслучая. 
СлучАй 1.1. $x M \geqslant y M$. По лемме 9 , а) имеем $\Phi(y M, l) \leqslant \Phi(x M, l)$. При этом $l \geqslant \mu+x y$, и тогда из (3.15) и (3.12) с использованием леммы 9, в) (монотонность отношения $\Phi(a, t) / t)$ получаем

$$
x_{M}^{\mu} y_{N}^{\delta} \leqslant M N+\left(\frac{\Phi(x M, \mu+x y)}{\mu+x y}-1\right)(|\delta-\varepsilon|+2 x y+M N+N M) .
$$

СлучАй 1.2. $x M<y M$. По лемме 9 , а) $\Phi(y M, l) \leqslant \Phi(x M, \mu+x y)$. Отсюда

$$
\begin{aligned}
\Phi(y M, l)-l & \leqslant \Phi(x M, \mu+x y)-\mu-x y+y M-x M \\
& \leqslant \Phi(x M, \mu+x y)-\mu-x y+y x .
\end{aligned}
$$

Вследствие неотрицательности числителя и знаменателя дроби $(l-s) / l$ и соотношений (3.12)-(3.14) имеем

$$
\frac{l-s}{l} \leqslant \frac{l-s+y M-x M}{l+y M-x M} \leqslant \frac{|\delta-\varepsilon|+x y+M N+N M}{\mu+x y} .
$$

Таким образом, второе слагаемое в правой части (3.15) можно оценить следующим образом:

$$
\begin{aligned}
& (\Phi(y M, l)-l) \frac{l-s}{l} \\
& \quad \leqslant\left(\frac{\Phi(x M, \mu+x y)}{\mu+x y}-1\right)(|\delta-\varepsilon|+x y+M N+N M)+x y \frac{l-s}{l} \\
& \quad \leqslant\left(\frac{\Phi(x M, \mu+x y)}{\mu+x y}-1\right)(|\delta-\varepsilon|+2 x y+M N+N M) .
\end{aligned}
$$

Здесь мы еще раз воспользовались соотношением (3.14), а затем (3.3). В результате, как легко видеть, снова приходим к оценке (3.16).

СлучАй 2. $y M+M N \geqslant \delta+y N$ и $y M \leqslant N M$. Тогда

$$
\begin{aligned}
x_{M}^{\mu} y_{N}^{\delta} & \leqslant x_{M}^{\mu} y+y y_{N}^{\delta} \leqslant x_{M}^{\mu} y+y N \leqslant x_{M}^{\mu} x+x y+y N \\
& \leqslant x M+\mu+x y+y N \leqslant x y+y M+\mu+x y+y N \leqslant \mu+2 x y+N M+y N .
\end{aligned}
$$

Ho

$$
\mu \leqslant \delta+|\varepsilon-\delta| \leqslant y M+M N-y N+|\varepsilon-\delta| \leqslant N M+M N-y N+|\varepsilon-\delta|,
$$

следовательно,

$$
\begin{aligned}
x_{M}^{\mu} y_{N}^{\delta} & \leqslant N M+M N+|\varepsilon-\delta|+2 x y+N M \\
& \leqslant M N+\left(\frac{\Phi(x M, t)}{t}-1\right)(|\delta-\varepsilon|+2 x y+2 M N)
\end{aligned}
$$

при любом $t>0$, в частности при $t=\mu$. 
СлучАЙ 3. $y M+M N \geqslant \delta+y N$ и $y M>N M$. Положим

$$
Q=\bigcup\{B(z, N M): z \in M\}
$$

- расширение множества $M$. Следуюшие факты проверяются элементарно: $Q$ выпукло; $N \subset Q ; y Q=y M-N M>0$;

$$
\begin{gathered}
x_{M}^{\mu} \subset Q \cap B(y, y x+x M+\mu) ; \\
y_{N}^{t} \subset Q \cap B(y, y N+t) \quad \forall t \geqslant 0 .
\end{gathered}
$$

Обозначим

$$
T=\chi(Q \cap B(y, y x+x M+\mu), Q \cap B(y, y N+0)) .
$$

Тогда для любого $\delta \geqslant 0$

$$
x_{M}^{\mu} y_{N}^{\delta} \leqslant \chi\left(x_{M}^{\mu}, y_{N}^{+0}\right) \leqslant T,
$$

и задача состоит в том, чтобы соответствующим образом оценить величину $T$. Рассмотрим 4 подслучая.

СлучАй 3.1. $y N<y x+x M+\mu, x M \geqslant y M-N M$. Применяя к величине $T$ оценку (2.24) и учитывая при этом первое из двух сделанных предположений, получаем

$$
T \leqslant \Phi(y Q, y x+x M+\mu-y Q)-(y x+x M+\mu-y N) \leqslant \Phi(y Q, p)-q,
$$

где $p=y x+x M+\mu-y Q, q=y x+x M+\mu-y N$.

По условию $y Q=y M-N M \leqslant x M$, значит,

$$
T \leqslant \Phi(x M, p)-q .
$$

Ho

$$
\begin{aligned}
\Phi(x M, p)-q & =M N+\Phi(x M, p)-p+p-q-M N \\
& \leqslant M N+\left(\frac{\Phi(x M, p)}{p}-1\right) p+p-q-M N \\
& \leqslant M N+\left(\frac{\Phi(x M, p)}{p}-1\right)(2 p-q-M N) .
\end{aligned}
$$

При этом

$$
p=y x+x M+\mu-y M+N M \geqslant \mu+N M .
$$

А так как $y M+M N \geqslant \delta+y N$, то

$$
2 p-q-M N=y x+x M+\mu-y M+2 N M+y N-(y M+M N) \leqslant|\varepsilon-\delta|+2 x y+2 N M .
$$

Таким образом,

$$
x_{M}^{\mu} y_{N}^{\delta} \leqslant T \leqslant M N+\left(\frac{\Phi(x M, \mu+N M)}{\mu+N M}-1\right)(|\delta-\varepsilon|+2 x y+2 N M) .
$$


СлучАЙ 3.2. $y N \leqslant y x+x M+\mu, x M<y M-N M$. Как и в предыдушем случае, получаем

$$
T \leqslant \Phi(y Q, p)-q
$$

с теми же $p$ и $q$. Поскольку $y Q=y M-N M>x M$, то по лемме 9 , а) имеем

$$
\Phi(y Q, p) \leqslant \Phi(x M, h),
$$

где $h=p+y Q-x M$. По аналогии с тем, как это проделано в предыдущем случае, можно получить следующую оценку:

$$
\Phi(x M, h)-q=M N+\left(\frac{\Phi(x M, h)}{h}-1\right)(2 h-q-M N),
$$

где

$$
h=p+y Q-x M \geqslant p \geqslant \mu+N M,
$$

а так как $y N \leqslant y M+M N-\delta$, то еще и оценку

$$
\begin{aligned}
2 h-q-M N & =y x+\mu+y N-M N-x M \\
& \leqslant y x+\mu+y M+M N-\delta-M N-x M \leqslant|\varepsilon-\delta|+2 x y .
\end{aligned}
$$

Следовательно,

$$
x_{M}^{\mu} y_{N}^{\delta} \leqslant M N+\left(\frac{\Phi(x M, \mu+N M)}{\mu+N M}-1\right)(|\delta-\varepsilon|+2 x y) .
$$

СлучАй 3.3. $y N \geqslant y x+x M+\mu, x M<y M-N M$. Первое условие с учетом неравенства (2.24) дает оценку

$$
T \leqslant \Phi(y Q, y N-y Q+0)-(y N-y x-x M-\mu)
$$

причем символ +0 можно убрать вследствие непрерывности $\Phi$. Так как $y Q=$ $y M-N M>x M$, то по лемме 9 , а) имеем

$$
\Phi(y Q, y N-y Q) \leqslant \Phi(x M, y N-x M) .
$$

Тогда, полагая $p=y N-x M, q=y N-y x-x M-\mu$, получаем

$$
T \leqslant \Phi(x M, p)-q \leqslant M N+\left(\frac{\Phi(x M, p)}{p}-1\right)(2 p-q-M N) .
$$

Поскольку

$$
p=y N-x M \geqslant x y+x M+\mu-x M=x y+\mu,
$$

а с учетом условия $y N \leqslant y M+M N-\delta$ еше и

$$
\begin{aligned}
2 p-q-M N & =y N-x M+y x+\mu-M N \\
& \leqslant y M+M N-\delta-x M+y x+\mu-M N \leqslant|\varepsilon-\delta|+2 x y,
\end{aligned}
$$

TO

$$
x_{M}^{\mu} y_{N}^{\delta} \leqslant M N+\left(\frac{\Phi(x M, \mu+x y)}{\mu+x y}-1\right)(|\delta-\varepsilon|+2 x y) .
$$


СлУчАЙ 3.4. $y N \geqslant y x+x M+\mu, x M \geqslant y M-N M$. Имеем

$$
T \leqslant \Phi(y Q, y N-y Q)-(y N-y x-x M-\mu) .
$$

Обозначим $p=y N-y Q, q=y N-y x-x M-\mu$. Так как $\Phi(y Q, p) \leqslant \Phi(x M, p)$, то для этих $p, q$ выполняется соотношение (3.17). При этом

$$
p=y N-y M+N M \geqslant y x+x M+\mu-y M+N M \geqslant \mu+N M .
$$

Кроме того, вследствие неравенства

$$
\mu \leqslant|\varepsilon-\delta|+\delta \leqslant|\varepsilon-\delta|+y M+M N-y N
$$

получаем

$2 p-q-M N=y N-2 y M+2 N M+y x+x M+\mu-M N \leqslant|\varepsilon-\delta|+2 x y+2 N M$.

Следовательно,

$$
x_{M}^{\mu} y_{N}^{\delta} \leqslant M N+\left(\frac{\Phi(x M, \mu+N M)}{\mu+N M}-1\right)(|\delta-\varepsilon|+2 x y+2 N M) .
$$

Итак, если учесть свойство монотонности отношения $\Phi(a, t) / t$, то в каждом из рассмотренных случаев получена оценка, уточняюшая (3.2). Значит, теорема 3 , а вместе с ней и теорема 1 полностью доказаны.

Безусловно, представляют интерес оценки $\tau$-устойчивости оператора $P$, не использующие информацию о геометрии единичной сферы пространства $X$. При этом можно ожидать, что их доказательство будет более простым, нежели доказательство теоремы 3 , а сами оценки - более сильньми, по сравнению с оценками, вытекаюшими из этой теоремы. Так, например, из теоремы 3 и соотношения $\Phi(a, t)-t \leqslant 2 a+t$ получаем неравенство

$$
\alpha\left(y_{N}^{\delta}, x_{M}^{\varepsilon}\right) \leqslant \alpha(N, M)+\left(\frac{2 x M}{\mu}+1\right)(|\delta-\varepsilon|+2 x y+2 \alpha(M, N)) .
$$

Его можно уточнить следующим образом.

Tеорема 4. Пусть $M$ - выпуклое подмножество из $X, N \subset X, x, y \in X$, $\varepsilon, \delta \geqslant 0, \mu+x y>0$, где $\mu=\max (\varepsilon, \delta)$. Тогда

$$
\alpha\left(y_{N}^{\delta}, x_{M}^{\varepsilon}\right) \leqslant \alpha(N, M)+\left(\frac{2 x M}{\mu+x y}+1\right)(|\delta-\varepsilon|+2 x y+M N+N M) .
$$


ДокАЗАТЕЛЬСтво. Сначала оценим величину $x_{M}^{\varepsilon} y_{N}^{\delta}$ через правую часть (3.18). При $y M+M N<\delta+y N$ эта оценка следует из неравенства (3.16), полученного при доказательстве теоремы 3 в случае 1. Пусть $y M+M N \geqslant y N+\delta$. Так как $y N-x M \leqslant x y+M N$, то

$$
\begin{aligned}
x_{M}^{\mu} y_{N}^{\delta} & \leqslant x_{M}^{\mu} y+y y_{N}^{\delta} \leqslant y x+x M+\mu+y N=y x+2 x M+\mu+(y N-x M) \\
& \leqslant 2 x y+2 x M+\mu+M N=M N+\left(\frac{2 x M}{\mu+2 x y}+1\right)(\mu+2 x y)
\end{aligned}
$$

и нужная оценка вытекает из соотношений

$$
\mu \leqslant|\varepsilon-\delta|+\delta \leqslant|\varepsilon-\delta|+y M+M N-y N \leqslant|\varepsilon-\delta|+N M+M N .
$$

Перейдем к оценке для $y_{N}^{\delta} x_{M}^{\varepsilon}$. В лемме 1 из [1] установлено неравенство

$$
y_{N}^{\delta} x_{M}^{\varepsilon} \leqslant N M+x_{M}^{\delta_{1}+0} x_{M}^{\varepsilon},
$$

где $\delta_{1}=\delta+x y+y N+N M-x M$. Отсюда получаем

$$
y_{N}^{\delta} x_{M}^{\varepsilon} \leqslant N M+x_{M}^{t+0} x_{M}^{\varepsilon}
$$

при $t=\mu+2 x y+M N+N M$. Поскольку $t>0$, то, как следует из [2, лемма 5], знак +0 в этом неравенстве можно опустить. Ввиду (2.9) имеем

$$
x_{M}^{t} x_{M}^{\varepsilon} \leqslant \frac{x_{M}^{t} x_{M}^{+0}}{t}(t-\varepsilon),
$$

и остается воспользоваться неравенством $x_{M}^{t} x_{M}^{+0} \leqslant 2 x M+t$ (заметим, что это же неравенство справедливо и для величины $x_{M}^{t+0} x_{M}^{+0}$, т.е. можно было бы обойтись и без леммы 5 из [2]). Теорема доказана.

Закончим параграф̆ поточечным вариантом оценки (1.8). Для $x \in X, M \in$ $\operatorname{Cv}(X), \varepsilon>0$ и $v=(x, M, \varepsilon)$ выполняются неравенства

$$
K_{\alpha}(v) \leqslant \frac{\Phi(x M, \varepsilon)}{\varepsilon}-1 \leqslant \frac{2 x M}{\varepsilon}+1 .
$$

\section{$\S 4$. Случай выпуклого множества $N$}

Нередко (например, в приложениях к теории экстремальных задач [9], [10]) выпуклыми являются оба множества $M$ и $N$. Естественно попытаться воспользоваться этой дополнительной информацией для уточнения полученных оценок устойчивости оператора $P$.

Обозначим $E=\min (x M, y N)$. Из теоремы 3 вытекает такое

СлЕДСТвИЕ 5. Если в теореме 3 дополнительно предположить выпуклость множества $N$, то будет выполняться неравенство

$$
\alpha\left(y_{N}^{\delta}, x_{M}^{\varepsilon}\right) \leqslant \alpha(N, M)+\left(\frac{\Phi(E, \mu)}{\mu}-1\right)(|\delta-\varepsilon|+2 x y+2 \alpha(M, N)) .
$$


ДоКАЗАТЕЛЬСТВО. Заметим, что ввиду сделанного предположения точки $(x, M, \varepsilon)$ и $(y, N, \delta)$ в теореме 3 можно поменять местами, получая таким образом еще одну оценку для $\alpha\left(y_{N}^{\delta}, x_{M}^{\varepsilon}\right)$, в которой величина $\Phi(x M, \mu)$ заменена на $\Phi(y N, \mu)$. Значит, в (3.2) при $\tau=\alpha$ вместо $\Phi(x M, \mu)$ можно поставить $\min [\Phi(x M, \mu), \Phi(y N, \mu)]$, причем вследствие монотонности функции $\Phi(a, t)$ по $a$ этот минимум равен $\Phi(E, \mu)$, что и требовалось.

Оценка (4.1) уточняет соответствующие результаты из [1], [9], [10], в которых не использовалась информация о геометрии единичной сферы пространства $X$. Более того, в отличие от оценки

$$
\alpha(P(v), P(w)) \leqslant\left(\frac{\Phi(E, \mu)}{\mu}-1\right) \varrho(v, w)
$$

вытекающей из (3.19), и ее следствия - неравенства

$$
\alpha(P(v), P(w)) \leqslant\left(\frac{2 E}{\mu}+1\right) \varrho(v, w),
$$

оценки из [9], [10] имеют качественно иной характер зависимости от входящих в них параметров: вместо $E$ стоит $\max (x M, y N)$, а вместо $\mu$ - величина $\min (\varepsilon, \delta)$.

Представляется естественным рассмотреть при $\tau=\alpha, \beta, \gamma$ следующие константы Липшица. Понимая, как и выше, под $E$ и $\mu$ соответственно $\min (x M, y N)$ и $\max (\varepsilon, \delta)$, положим

$$
\begin{array}{r}
\widetilde{\mathscr{V}}(a, s)=\{(v, w) \in \mathscr{V} \times \mathscr{V}: v=(x, M, \varepsilon), w=(y, N, \delta), \\
M, N \in \operatorname{Cv}(X), E \leqslant a, \mu \geqslant s, \varrho(v, w) \neq 0\}, \\
\widetilde{\mathscr{V}}^{\prime}(a, s)=\{(v, w) \in \widetilde{\mathcal{V}}: E=a, \mu=s\}, \\
\widetilde{K}_{\tau}(a, s)=\sup \left\{\frac{\tau(P(w), P(v))}{\varrho(v, w)}:(v, w) \in \widetilde{\mathcal{V}}(a, s)\right\}, \\
\widetilde{K}_{\tau}^{\prime}(a, s)=\sup \left\{\frac{\tau(P(w), P(v))}{\varrho(v, w)}:(v, w) \in \widetilde{\mathcal{V}}^{\prime}(a, s)\right\} .
\end{array}
$$

Ввиду симметрии очевидны равенства

$$
\begin{aligned}
\widetilde{K}_{\alpha}(a, s) & =\widetilde{K}_{\beta}(a, s)=\widetilde{K}_{\gamma}(a, s), \\
\widetilde{K}_{\alpha}^{\prime}(a, s) & =\widetilde{K}_{\beta}^{\prime}(a, s)=\widetilde{K}_{\gamma}^{\prime}(a, s) .
\end{aligned}
$$

Кроме того,

$$
\begin{gathered}
\widetilde{K}_{\tau}^{\prime}(a, s) \leqslant \widetilde{K}_{\tau}(a, s), \\
\widetilde{K}_{\tau}(a, s)=\sup \left\{\widetilde{K}_{\tau}^{\prime}(b, t): b \leqslant a, t \geqslant s\right\} .
\end{gathered}
$$

Из оценок (4.2), (4.3) и примера $1 \S 3$ из [2] вытекает следуюшая 
Tеорема 5. При $a \geqslant 0, s>0$ имеем

$$
\widetilde{K}_{\alpha}(a, s) \leqslant \frac{\Phi(a, s)}{s}-1 \leqslant \frac{2 a}{s}+1 .
$$

А если дополнительно предположить, что единичная сфера пространства $X$ содержит отрезок длины 2 , то

$$
\widetilde{K}_{\alpha}^{\prime}(a, s)=\widetilde{K}_{\alpha}(a, s)=\frac{2 a}{s}+1
$$

В заключение сделаем одно общее замечание. Нетрудно показать, что в теоремах 1-4 множество $M$, а в теореме 5 и множество $N$ можно считать "относительно выпуклыми", т.е. такими, замыкание которых в $X$ выпукло.

\section{§5. Приложения к оценкам устойчивости непрерывного селектора оператора $P$}

Селектором многозначного отображения $F(x)$ называется такое однозначное отображение $s(x)$, для которого $s(x) \in F(x)$ при любом $x$. В последнее время большое внимание уделяется вопросам сушествования непрерывного и липшицева селектора оператора метрического проектирования $x \mapsto x_{M}$ (см., например, [13], [19], [20] и библиографию к ним). В [21]-[23] изучался непрерывньй селектор оператора $x \mapsto x_{M}^{\varepsilon}$ как для выпуклых, так и для невыпуклых $M$. В [24] получены оценки устойчивости непрерывного селектора оператора $P:(x, M, \varepsilon) \mapsto$ $x_{M}^{\varepsilon}$ c $M \in \operatorname{Cv}(X)$.

В настояшем параграфе уточняются оценки работы [24]. Пусть $Y$ - банахово подпространство из $X$. Положим

$$
\mathscr{V}_{Y}=\{(x, M, \varepsilon) \in \mathscr{V}(X): M \in \operatorname{Clv}(Y), \varepsilon>0\}
$$

где $\operatorname{Clv}(Y)$ - множество всех непустых замкнутых выпуклых подмножеств из $Y$. Далее $\mathscr{V}_{Y}$ рассматривается как метрическое пространство $\left(\mathscr{V}_{Y}, \varrho\right)$ с метрикой $\varrho$, индуцированной из $\mathscr{V}$.

Рассмотрим $P_{Y}$ - сужение $P$ на $\mathscr{V}_{Y}$. Как отмечено в [24], из $\alpha$-непрерьвности оператора $P_{Y}$ и теоремы Майкла [25] немедленно следует сушествование непрерывного селектора $s(v)$ оператора $P_{Y}$. Кроме того, в [24] показано, что характер непрерывности селектора $s(v)$ зависит от выбора подпространства $Y$. Так, если $Y$ - конечномерное подпространство, то с помощью точек Штейнера и оценки (4.3) строится поточечно липшицев селектор, а если $Y$ - банахово подпространство, допускающее перенормировку в равномерно выпуклое пространство $\left(Y,\|\cdot\|_{0}\right)$, то опять же с использованием (4.3) вьписывается оценка устойчивости селектора через модуль выпуклости пространства $\left(Y,\|\cdot\|_{0}\right)$. Естественно теперь воспользоваться более точной оценкой (4.2).

Сначала предположим, что банахово подпространство $Y$ из $X$ имеет эквивалентную норму $\|\cdot\|_{0}$ с модулем выпуклости $\varphi_{0}(t)$. Пусть $\nu$ - мера перенормировки 
пространства $Y$, т.е. такое минимальное число $\nu \geqslant 1$, для которого вьполняются неравенства

$$
\nu^{-1}\|y\|_{0} \leqslant\|y\| \leqslant \nu\|y\|_{0}
$$

при любом $y \in Y$. Для $a, t \geqslant 0, a+t>0$, положим

$$
\Lambda[a, t]=(a+t) \varphi_{0}^{-1}\left(\frac{2 t}{a+t}\right) .
$$

В [24] доказано, что $\Lambda[a, t]$ не убывает по каждой из переменных.

Теорема 6. Пусть $Y$ - банахово подпространство из $X$ (возможсно, совпадающее с самим $X$ ), допускающее әквивалентную перенормировку в равномерно выпуклое пространство $\left(Y,\|\cdot\|_{0}\right)$ с модулем выпуклости $\varphi_{0}(t) u$ мерой перенормировки $\nu$. Тогда оператор $P_{Y}$ обладает на множестве $\mathscr{V}_{Y}$ непрерывным селектором $s(v)$, удовлетворяющим при любых $v=(x, M, \varepsilon)$, $w=(y, N, \delta) \in \mathscr{V}_{Y}$ следующим неравенствам:

$$
\|s(v)-s(w)\| \leqslant \nu^{2} \Lambda\left[G, \alpha\left(x_{M}^{\varepsilon}, y_{N}^{\delta}\right)\right] \leqslant \nu^{2} \Lambda\left[G,\left(\frac{\Phi(E, \mu)}{\mu}-1\right) \varrho(v, w)\right]
$$

$2 \partial e$

$$
\begin{gathered}
G=\min (\|x\|+x M,\|y\|+y N), \\
E=\min (x M, y N), \quad \mu=\max (\varepsilon, \delta) .
\end{gathered}
$$

Здесь и далее, как обычно, функция $\Phi(a, s)$ определяется через модуль выпуклости $\varphi$ пространства $X$.

Первое неравенство в (5.1) фактически доказано в [24], а второе является следствием (4.2) и свойства монотонности функции $\Lambda[a, t]$ по $t$. При этом имеется в виду то, что, как и в [24], в качестве $s(v)$ берется проекция нуля на множество $x_{M}^{\varepsilon}$ в пространстве $\left(Y,\|\cdot\|_{0}\right)$. Отметим, что в качестве $G$ можно взять величину $2 \min (\|x\|,\|y\|)$, которая, очевидно, не меньше, чем $G$.

Рассмотрим теперь случай конечномерного подпространства $Y \subset X, \operatorname{dim} Y=n$. Селектор $s(v)$ назовем однородным, если для любого $t \in \mathbb{R}$ выполняется равенство $s(t v)=t s(v)$, где для $v=(x, M, \varepsilon)$ произведение $t v$ определяется как точка $(t x, t M,|t| \varepsilon)$. Согласно теореме Джона (см., например, [26]) пространство $(Y,\|\cdot\|)$ можно перенормировать в евклидово $\left(Y,\|\cdot\|_{2}\right)$ так, чтобы при любых $x \in Y$ выполнялись неравенства

$$
\|x\| \leqslant\|x\|_{2} \leqslant \sqrt{n}\|x\| .
$$

Известно [27], что точки Штейнера $s(A)$ выпуклых ограниченных множеств $A \subset Y$ удовлетворяют условию Липшища относительно хаусдорфовой метрики $\alpha_{2}(A, B)$ в пространстве $\left(Y,\|\cdot\|_{2}\right)$ с константой Липшица, равной $2 \omega_{n-1} / \omega_{n}$, где $\omega_{n}$ - объем единичного шара в $n$-мерном пространстве $\left(Y,\|\cdot\|_{2}\right), n \geqslant 2$.

ЛЕММА 11. $2 \omega_{n-1} / \omega_{n}<\sqrt{n} n p u n \geqslant 2$. 
ДокАЗАТЕЛЬСТво. Воспользуемся формулами $\omega_{n}=\pi^{n / 2} / \Gamma(1+n / 2)$, $\Gamma(n+1)=n !, \quad \Gamma(n+1 / 2)=\sqrt{\pi}(2 n) ! 4^{n} n !$. Рассмотрим случай $n=2 k$. По формуле Стирлинга получаем

$$
\frac{2 \omega_{2 k-1}}{\omega_{2 k}}=\frac{2 k ! 4^{k} k !}{\pi(2 k) !}=2 \sqrt{\frac{k}{\pi}} e^{\theta_{k} /(6 k)-\theta_{k}^{\prime} /(24 k)},
$$

где $0<\theta_{k}, \theta_{k}^{\prime}<1$. Следовательно,

$$
\frac{2 \omega_{2 k-1}}{\omega_{2 k}}<2 \sqrt{\frac{k}{\pi}} e^{1 / 6}<\sqrt{n}
$$

Аналогично, при $n=2 k+1$ имеем

$$
\frac{2 \omega_{2 k}}{\omega_{2 k+1}}<2 \sqrt{\frac{k}{\pi}} e^{1 / 24}<\sqrt{n}
$$

Лемма доказана.

Итак, константа Липшица проектора Штейнера меньше $\sqrt{n}$ при $n \geqslant 2$ и, очевидно, равна 1 при $n=1$. Таким образом, для любых выпуклых ограниченных множеств $A, B \subset Y$ справедливо неравенство

$$
\|s(A)-s(B)\|_{2} \leqslant \sqrt{n} \alpha_{2}(A, B) .
$$

Отсюда и из (5.2) получаем

$$
\|s(A)-s(B)\| \leqslant\|s(A)-s(B)\|_{2} \leqslant n \alpha(A, B),
$$

где $\alpha(A, B)$ - хаусдорфово расстояние в $(Y,\|\cdot\|)$. Если теперь при доказательсве теоремы 4 из [24] воспользоваться этой оценкой, а также неравенством (4.2) вместо (4.3), то придем к такому результату.

Tеорема 7. Пусть $Y-n$-мерное подпространство из $X$; тогда для оператора $P_{Y}$ существует однородный селектор s, удовлетворяющий неравенствам

$$
\|s(v)-s(w)\| \leqslant n \alpha\left(x_{M}^{\varepsilon}, y_{N}^{\delta}\right) \leqslant n\left(\frac{\Phi(E, \mu)}{\mu}-1\right) \varrho(v, w),
$$

əде $v, w, E, \mu-$ те же, что и в теореме 6.

Как и в [24], в качестве значения $s(v)$ берется точка Штейнера множества $x_{M}^{\varepsilon}$ в пространстве $\left(Y,\|\cdot\|_{2}\right)$. Определение и свойства точек Штейнера можно найти в [26], [28].

Заметим, что оценка (5.3) отличается от соответствующей оценки теоремы 4 из [24] еше и тем, что вместо множителя $n \nu^{2}$ удалось поставить $n$. 
Из теоремы 7 немедленно вытекают оценки для констант Липшица селектора $s(v)$. Положим

$$
\begin{gathered}
k(v)=\sup \left\{\frac{\|s(v)-s(w)\|}{\varrho(v, w)}: w \in \mathscr{V}_{Y}, \varrho(v, w) \neq 0\right\} ; \\
\widetilde{\mathscr{V}}_{Y}(a, s)=\left\{(v, w) \in \mathscr{V}_{Y} \times \mathscr{V}_{Y}: v=(x, M, \varepsilon), w=(y, N, \delta),\right. \\
E \leqslant a, \mu \geqslant s, \varrho(v, w) \neq 0\} ; \\
\widetilde{k}(a, s)=\sup \left\{\frac{\|s(v)-s(w)\|}{\varrho(v, w)}:(v, w) \in \widetilde{\mathscr{V}}_{Y}(a, s)\right\} .
\end{gathered}
$$

СЛЕДСТвИЕ 6 . Для $v=(x, M, \varepsilon) \in \mathscr{V}_{Y} u a \geqslant 0, s>0$ имеем

$$
k(v) \leqslant n\left(\frac{\Phi(x M, \varepsilon)}{\varepsilon}-1\right), \quad \tilde{k}(a, s) \leqslant n\left(\frac{\Phi(a, s)}{s}-1\right) .
$$

\section{§6. Константы Липшица по отдельным переменным}

При доказательстве равенств (1.9) из теоремы 1 фактически было показано (см. конец $\S 1$ ), что оценки $\tau$-констант Липшица оператора $P(x, M, \varepsilon)$ по совокупности трех переменных являются в обшем случае неулучшаемыми оценками и для $\tau$-констант Липшица оператора $P$ при вариации одной переменной $\varepsilon$. Естественно поставить вопрос о неулучшаемых оценках $\tau$-констант Липшица по двум другим переменным $x$ и $M$. Оказывается, что оценки устойчивости оператора $P$ по совокупности трех переменных, полученные в $\S 2,3$, приводят к неулучшаемым оценкам $\tau$-констант Липшица не только по переменной $\varepsilon$, но и по $x$ и $M$. Определим $\tau$-константы Липшица, $\tau=\alpha, \beta, \gamma$, в точке $v=(x, M, \varepsilon)$ по каждой из переменных в отдельности следуюшим образом:

$$
\begin{aligned}
& K_{\tau}^{1}(v)=\sup \left\{\frac{\tau\left(y_{M}^{\varepsilon}, x_{M}^{\varepsilon}\right)}{x y}: y \in X, y \neq x\right\}, \\
& K_{\tau}^{2}(v)=\sup \left\{\frac{\tau\left(x_{N}^{\varepsilon}, x_{M}^{\varepsilon}\right)}{\alpha(M, N)}: \varnothing \neq N \subset X, \alpha(M, N) \neq 0\right\}, \\
& K_{\tau}^{3}(v)=\sup \left\{\frac{\tau\left(x_{M}^{\delta}, x_{M}^{\varepsilon}\right)}{|\delta-\varepsilon|}: \delta \geqslant 0, \delta \neq \varepsilon\right\} .
\end{aligned}
$$

Для $a, s \geqslant 0, i=1,2,3$, обозначим

$$
K_{\tau}^{i}(a, s)=\sup \left\{K_{\tau}^{i}(v): v=(x, M, \varepsilon) \in \mathscr{V}, M \in \operatorname{Cv}(X), x M \leqslant a, \varepsilon \geqslant s\right\}
$$

Ясно, что при $i=1,2,3$ выполняются соотношения

$$
K_{\alpha}^{i}(v)=\max \left(K_{\beta}^{i}(v), K_{\gamma}^{i}(v)\right), \quad K_{\alpha}^{i}(a, s)=\max \left(K_{\beta}^{i}(a, s), K_{\gamma}^{i}(a, s)\right) .
$$


Через $l_{\infty}^{n}$ обозначим пространство векторов $x=\left(x_{1}, \ldots, x_{n}\right) \in \mathbb{R}^{n}$ с нормой $\|x\|_{\infty}=\max \left|x_{i}\right|$. Заметим, что единичная сфера $S$ пространства $X$ содержит отрезок длины 2 тогда и только тогда, когда пространство $l_{\infty}^{2}$ изометрично и изоморфно вкладывается в $X$.

TeOpema 8. 1) При $a \geqslant 0, s>0$ имеем

$$
\begin{aligned}
& K_{\alpha}^{1}(a, s) \leqslant 2\left(\frac{\Phi(a, s)}{s}-1\right) \leqslant \frac{4 a}{s}+2 \\
& K_{\alpha}^{2}(a, s) \leqslant 2\left(\frac{\Phi(a, s)}{s}-1\right)+1 \leqslant \frac{4 a}{s}+3 \\
& K_{\alpha}^{3}(a, s) \leqslant \frac{\Psi(a, s)}{s} \leqslant \frac{2 a}{s}+1 .
\end{aligned}
$$

2) Если дополнительно предположить, что единичная сфера $S$ пространства X содержит отрезок длины 2, то при $\tau=\alpha, \beta, \gamma$ справедливы равенства

$$
\begin{aligned}
& K_{\tau}^{2}(a, s)=\frac{4 a}{s}+3 \\
& K_{\tau}^{3}(a, s)=\frac{2 a}{s}+1 .
\end{aligned}
$$

3) Если же в пространство $X$ изометрично и изоморфно вкладывается пространство $l_{\infty}^{3}$, то при $\tau=\alpha, \beta, \gamma$

$$
K_{\tau}^{1}(a, s)=\frac{4 a}{s}+2
$$

ДоКАЗАТЕЛЬСТво. Оценки (6.1) сразу следуют из (1.8) - нужно только учесть, что величина $x y$ входит в формулу для расстояния $\varrho(v, w)$ с коэффициентом 2. Оценки (6.2) вытекают из неравенства

$$
\alpha\left(x_{N}^{s}, x_{M}^{s}\right) \leqslant\left[2\left(\frac{\Phi(x M, s)}{s}-1\right)+1\right] \alpha(M, N)
$$

которое, в свою очередь, является частным случаем оценки (3.2). Из (2.9) с учетом (2.3) и теоремы 2 без труда получаем неравенство (6.3). По поводу равенства (6.5) см. конец $\S 1$. Доказательство (6.4), очевидно, достаточно провести только в случае $X=l_{\infty}^{2}$, чем мы и воспользуемся.

Пусть $x$ - начало координат $(0,0)$ и $t>0$. За множество $M$ возьмем отрезок с конщами $p=(-a-s, a+s+2 t), q=(a, a)$, а за $N$-отрезок с концами $k=(-a-$ $s-t, a+s+t), l=(a+t, a+t)$. Ясно, что $x M=x q=a$, а так как $p k=q l=t$, то $\alpha(M, N)=t$. Кроме того, $x_{N}^{s}=N$. Обозначим через $z$ точку пересечения $M \cap S(x, x M+s)$; тогда $x_{M}^{s}=[z, q]$. Поэтому при $\tau=\alpha, \beta$ имеем

$$
\tau\left(x_{N}^{s}, x_{M}^{s}\right)=k z .
$$


Положим $c=(-a-s, a+s), d=(-a-s, a)$. Тогда можно записать $k z=k c+$ $c z=t+c z$. Но по построению $c z / d q=p c / p d$, где $d q=2 a+s, p c=2 t, p d=s+2 t$. Значит,

$$
k z=t+\frac{2 a+s}{s+2 t} 2 t
$$

Отсюда для $\tau=\alpha, \beta$ получаем

$$
K_{\tau}^{2}(a, s) \geqslant \sup _{t>0} \frac{\tau\left(x_{N}^{s}, x_{M}^{s}\right)}{t}=1+\sup _{t>0} \frac{4 a+2 s}{s+2 t}=\frac{4 a}{s}+3,
$$

что и доказывает (6.4) при $\tau=\alpha, \beta$. Рассмотрим случай $\tau=\gamma$.

Поменяем местами множества $M$ и $N: M=[k, l], N=[p, q]$. Затем во всех координатах построенных выше точек заменим параметр $a$ на $a-t$. Тогда будем иметь $x M=a, d q=2 a+s-2 t, p c, p d-$ те же, что и раньше. По аналогии с предыдушим случаем получаем

$$
\gamma\left(x_{N}^{s}, x_{M}^{s}\right)=k z=t+\frac{2 a+s-2 t}{s+2 t} 2 t,
$$

откуда

$$
K_{\gamma}^{2}(a, s) \geqslant \sup _{t>0} \frac{\gamma\left(x_{N}^{s}, x_{M}^{s}\right)}{t}=\frac{4 a}{s}+3 .
$$

Осталось доказать равенство (6.6). Опять замечаем, что его достаточно установить только в случае $X=l_{\infty}^{3}$. Пусть $x=(0,0,0), t>0, p=(-a, a, a)$, $q=(a+s+2 t, a+s+2 t, a), M=\operatorname{span}\{p, q\}$ - прямая, проходящая через точки $p$ и $q$. Имеем $x M=x p=a$. Через $z$ обозначим точку пересечения $[p, q] \cap S(x, a+s)$. Понятно, что $x_{M}^{s}=[p, z]$. Положим $y=(t, t,-t)$. Нетрудно проверить, что $y M=a+t$, а так как $y q=y M+s$ и $y p=y M$, то $y_{M}^{s}=[p, q]$. Таким образом,

$$
y_{M}^{s} x_{M}^{s}=q z \text {. }
$$

Пусть $c=(a+s+2 t, a+s, a), d=(a+s+2 t, a, a)$. Тогда по построению $q z / q p=q c / q d$, причем $q p=2 x M+s+2 t, q c=2 t, q d=s+2 t$. Значит,

$$
K_{\beta}^{1}(a, s) \geqslant \sup _{t>0} \frac{y_{M}^{s} x_{M}^{s}}{t}=2 \sup _{t>0} \frac{2 a+s+2 t}{s+2 t}=\frac{4 a}{s}+2
$$

что и доказывает равенство (6.6) при $\tau=\beta$.

Для его доказательства при $\tau=\gamma$ нужно в построенном примере поменять местами точки $x$ и $y$, считая при этом, что $x M=a$, т.е., другими словами, производя всюду замену $a+t$ на $t$. Тогда $y M=a-t, p q=2 y M+s+2 t=2 a+s, q c, q d-$ те же, что и вьше. А так как

$$
\gamma\left(y_{M}^{s}, x_{M}^{s}\right)=x_{M}^{s} y_{M}^{s}=q z=q p \cdot q c / q d,
$$

то

$$
K_{\gamma}^{1}(a, s) \geqslant \sup _{t>0} \frac{4 a+2 s}{s+2 t}=\frac{4 a}{s}+2 .
$$

Теорема доказана. 
ЗАмЕЧАНИЕ 1. Используя (2.22) можно выписать более точные, чем (6.1), (6.2), оценки для $K_{\beta}^{i}(a, s)$, заменяя величину $\Phi(a, s) / s-1$ на $\Psi(a, s) / s$.

ЗАмЕчАнИЕ 2 . По аналогии с константами $\widetilde{K}_{\tau}(a, s)$ можно ввести (с соответствуюшими упрощениями в определениях) константы $\widetilde{K}_{\tau}^{i}(a, s), i=1,2,3$, для которых также будут выполняться соотношения (6.1)-(6.6) теоремы 8. Это становится вполне очевидным, если учесть, что во всех примерах, построенных при доказательстве теоремы 8 , фигурируют только выпуклые аппроксимируюшие множества. Не менее очевиден и другой вариант теоремы 8 , когда константы $K_{\tau}^{i}(a, s)$ заменяются на ${K^{\prime}}_{\tau}^{i}(a, s)$, аналогичные константам $K_{\tau}^{\prime}(a, s)$; при этом все утверждения теоремы переносятся в полной мере.

\section{Список литературы}

1. Маринов A.B. Устойчивость $\varepsilon$-квазирешений операторных уравнений I рода // Приближение функций полиномами и сплайнами: Сб. ст. Свердловск: УНЦ АН СССР, 1985. C. $105-117$.

2. Маринов $A$. B. Оценки устойчивости метрической $\varepsilon$-проекции через модуль выпуклости пространства // Тр. ИММ УрО РАН. 1992. Т. 2. С. 85-109.

3. Wulbert D. E. Continuity of metric projections. Approximation theory in a normed linear lattice: Thes. Univ. Texas Comp. Center. Austin, 1966. P. 105.

4. Лисковец О.А. Метод $\varepsilon$-квазирешений для уравнений I рода // Дифференц. уравн. 1973. T. 9. №10. C. 1851-1861.

5. Лисковец О.А. Вариационные методы решения неустойчивых задач. Минск: Наука и техника, 1981.

6. Бердышев В. И. Варьирование нормы в задаче о наилучшем приближении // Матем. заметки. 1981. Т. 29. №2. С. 181-196.

7. Бердышев В.И. Непрерывность многозначного отображения, связанного с задачей минимизации функционала // Изв. АН СССР. Сер. матем. 1980. Т. 44. № 3. С. 483-509.

8. Маринов A. В. Непрерывность и связность метрической $\delta$-проекции // Аппроксимация в конкретных и абстрактных банаховых пространствах: Сб. ст. Свердловск: УНЦ АН CCCP, 1987. C. 82-95.

9. Attouch H., Wets R.J.-B. Lipschitsian stability of $\varepsilon$-approximate solutions in convex optimisation. Laxenburg, 1987. 31 p. (WP-87-25, IIASA).

10. Attouch H., Wets R.J.-B. Quantitative stability of variational systems. I. The epigraphical distance. Laxenburg, 1988. 41 p. (WP-88-8, IIASA).

11. Гаркави А. Л. Теория наилучшего приближения в линейных нормированных пространствах // Итоги науки и техники. Матем. анализ. М.: ВИНИТИ, 1969. С. 75-132.

12. Власов Л. П. Аппроксимативные свойства множеств в линейных нормированных пространствах // УМН. 1973. Т. 28. №6. С. 3-66.

13. Deutsch F. A survey of metric selections // Contemporery Math. 1983. V. 18. P. 49-71.

14. Figiel T. On the moduli of convexity and smoothness // Studia Math. 1976. V. LVI. № 2. P. 121-155.

15. Гурарий В.И. О дифференциальных свойствах модулей выпуклости банаховых пространств // Математические исследования. Т.ІІ. Вып. 1. Кишинев: РИО АН МССР, 1966. C. $141-148$.

16. Дистель Дж. Геометрия банаховых пространств. Киев: Вища шк., 1980.

17. Björnestål B. O. Local Lipschitz continuity of the metric projection operator // Banach Center Publications. 1979. V. 4. P. 43-54. 
18. Кадец М.И. О топологической эквивалентности равномерно выпуклых пространств // УМН. 1955. Т. 10. № 4. С. 137-141.

19. Deutsch F., Li W., Park S.-H. Characterization of continuous and Lipschitz continuous metric selections in normed linear spaces // J. Approxim. Theory. 1989. V. 58. № 3 . P. 297-314.

20. Brown A. L. Set valued mappings, continuous selections, and metric projections // J. Approxim. Theory. 1989. V. 57. № 1. P. 48-68.

21. Конягин C.В. О непрерывных операторах обобщенного рационального приближения // Матем. заметки. 1988. Т. 44. № 3. С. 404.

22. Царьков И. Г. Свойства множеств, обладающих непрерывной выборкой из оператора $P^{\delta}$ // Матем. заметки. 1990. Т. 48. № 4. С. 122-131.

23. Альбрехт П. В. Порядки модулей непрерывности операторов почти наилучшего приближения // Матем. сб. 1994. Т. 185. №9. С. 3-28.

24. Маринов A.B. Оценки устойчивости непрерывной селекции для метрической почти-проекции // Матем. заметки. 1994. Т. 55. № 4. С. 47-53.

25. Michael E. Continuous selections // J. Ann. Math. Ser. 2. 1956. V. 63. № 2. P. 361-381.

26. Грюнбаум Б. Этюды по комбинаторной геометрии и теории выпуклых тел. М.: Наука, 1971.

27. Посицельский Е. Д. О липшицевых отображениях в пространстве выпуклых тел // Оптимизация. Сб. трудов. 1971. № 4 (21). С. 83-89.

28. Shepard G.C. The Steiner point of a convex polytope // Canad. J. Math. 1966. V. 18. № 6. P. 1294-1300.

E-mail: marinov@apprecat.imm.intec.ru

Поступило в редакцию

30.VIII.1995 